\title{
Prediction of the Distribution of Perennial Cultivated Grasses in the Northwest Sichuan Plateau, China under Climate Change
}

\author{
Bin Guo ${ }^{1,2}$, San Wang1,2, Chao Chen ${ }^{1,3^{*}}$, Mingtian Wang1,3,4, Tingting $\mathrm{Li}^{5}$ \\ ${ }^{1}$ Chengdu/Heavy Rain and Drought-Flood Disasters in Plateau and Basin Key Laboratory of Sichuan Province, \\ Institute of Plateau Meteorology, China Meteorological Administration, Chengdu, China \\ ${ }^{2}$ Aba Prefecture Meteorological Administration, Maerkang, China \\ ${ }^{3}$ Provincial Key Laboratory of Water-Saving Agriculture in Hill Areas of Southern China, Chengdu, China \\ ${ }^{4}$ Sichuan Meteorlogical Observatory, Chengdu, China \\ ${ }^{5}$ State Key Laboratory of Atmospheric Boundary Layer Physics and Atmospheric Chemistry, Institute of Atmospheric Physics, \\ Chinese Academy of Science, Beijing, China \\ Email: *Chenchao16306@sina.com
}

How to cite this paper: Guo, B., Wang, S., Chen, C., Wang, M. T., \& Li, T. T. (2019). Prediction of the Distribution of Perennial Cultivated Grasses in the Northwest Sichuan Plateau, China under Climate Change. Journal of Geoscience and Environment Protection, 7, 95-111

https://doi.org/10.4236/gep.2019.710008

Received: August 20, 2019

Accepted: October 22, 2019

Published: October 25, 2019

Copyright $\odot 2019$ by author(s) and Scientific Research Publishing Inc. This work is licensed under the Creative Commons Attribution International License (CC BY 4.0).

http://creativecommons.org/licenses/by/4.0/ (c) (i) Open Access

\begin{abstract}
In order to determine the threshold and potential distribution of climatic conditions for perennial cultivated grasses in the Northwest plateau of Sichuan, China, the niche model is used to simulate the main climatic factors affecting the distribution of perennial cultivated grasses, and to predict the current and future climate change prospects. The results show that: 1) Wet index, accumulated temperature, isothermal, and annual average temperature range are the four major climatic factors affecting the distribution of perennial cultivated grasses in the Northwest Sichuan Plateau. 2) Model training and prediction data can be achieved well under the appropriate range of major climatic factors. 3) Under the background of climate warming, from now to 2080, the potential distribution of perennial cultivated grasses in the Northwest plateau of Sichuan, China will increase. The study aims to provide scientific decision-making basis for maintaining grassland ecosystem stability and promoting desertification management and even industrial restructuring of agriculture and animal husbandry.
\end{abstract}

\section{Keywords}

Clmatei Change, MaxEnt Model, Dominant Factor, Perennial Cultivated Grasses, Northwest Sichuan Plateau

\section{Introduction}

The IPCC's fifth assessment report pointed out that global warming has become 
a reality in the past 130 years (Qin \& Stocker, 2014; Yu et al., 2003), and the warming trend has led to a sharp increase in temperature-related extreme events in China. Ding et al. (2006) found that the warming of the Qinghai-Tibet Plateau of China is more significant than that of other parts of the country, especially in the main part of the plateau. Climate is one of the main factors determining the distribution of biomes. Global warming has a profound impact on the geographical distribution of biomes and the distribution characteristics of suitable areas (Davis et al., 2001; Bellard et al., 2012).

The maximum entropy model (MaxEnt), as a spatial distribution model of species geographical scale, has been widely used in the prediction of potential distribution areas of species under the background of climate change (Zhang, 2015). Chen et al. (2019) used the MaxEnt model to analyze the suitable climatic characteristics of Leymus chinensis in China. Hu et al. (2015b) simulated the distribution pattern of Stipa breviflora in the history, current and future of the Qinghai-Tibet Plateau, and explored the causes of changes in species distribution. Yang (2016) constructed a climatically suitable distribution area of different grades of Stipa genus by the maximum entropy model and ArcGIS spatial analysis. Chen et al. (2016) predicted the current distribution of Stipa breviflora and its potential distribution in China in 2050. Liu et al. (2018) simulated the main climatic factors affecting the distribution of Carex lasiocarpa, and predicted the distribution pattern of Carex lasiocarpa under historical and future climate scenarios. However, there are few analyses on the suitable grassland cultivation areas in the Northwest Sichuan Plateau. The research mainly focuses on the investigation of wild forage resources community, cultivated grass selection, genetic diversity, hydrothermal ecological characteristics, etc. (Ma et al., 2014; Tang et al., 2018; Zhang, 2007; Pan \& Wang, 1993; Wang et al., 1995; Zhang et al., 2018).

The Sichuan Plateau is part of the Qinghai-Tibet Plateau, one of the five largest pastoral areas in China and the largest animal husbandry base in Sichuan. Located in the Ganzi Tibetan Autonomous Prefecture and the Aba Tibetan and Qiang Autonomous Prefecture in the Northwest part of Sichuan Province, China, the terrain is complex. Most of the areas are above $3000 \mathrm{~m}$ above sea level. The three-dimensional change of climate is obvious. It is an important animal husbandry and forestry base. The Yangtze River and the Yellow River are important ecological barrier upstreams (Zhang, 2014). In recent years, natural grassland in this area has been degraded, and grassland animals have been overloaded with animal husbandry (Zhang \& Zhang, 2013). It is even more important to carry out fine pasture introduction and artificial grassland establishment. After investigation, the local government established a number

\section{Materials and Methods}

\subsection{Determination of Distribution Data of Cultivated Grasses in the Northwest Sichuan Plateau}

This study is based on the collection of a large amount of literature (Ma et al., 
2014; Hong, 1989; Department of Animal Husbandry and Veterinary, Ministry of Agriculture, National Animal Husbandry and Veterinary Station, 1996; Duo, 1986; Dong, 1986), grassland scientific research management department pasture planting expert experience, field survey data (https://www.zhiwutong.com), China Digital Herbarium (http://www.cfh.ac.cn), plants Tong species database, China Flora (http://frps.iplant.cn/sheng) and other websites open data, collected and compiled 45 samples of cultivated grasses in the Northwest Sichuan Plateau (Figure 1).

In addition to the artificially planted pasture base using GPS to obtain latitude and longitude coordinate information, the remaining records do not provide latitude and longitude coordinate information because of only the location description. In this case, the suitable area is established by expert scoring, and the latitude and longitude information is obtained by using Google Earth.

\subsection{Environmental Factor Data Collection and Screening}

The Chinese meteorological background data set is downloaded from the Environmental Science Data Center of the Chinese Academy of Sciences

(http://www.resdc.cn). Including: spatial average resolution (Ta), average precipitation ( $\mathrm{Pa}), \geq 0^{\circ} \mathrm{C}$ accumulated temperature, $\geq 10^{\circ} \mathrm{C}$ accumulated temperature, dryness and wetting index (IM, Thornthwaite method (1948)) spatial distribution data set. The dryness index has been expanded by 1000 times, the wetness index has been expanded by 100 times, and the remaining climatic factors have been expanded by 10 times; the Sichuan Meteorological Bureau issued the DEM data of the $90 \mathrm{~m}$ resolution of the geodetic coordinates of Sichuan Province, the city-level boundary vector data, $30 \mathrm{~m}$ Cover surface coverage data; Bioclimate data from 1950 to 2000 (2000s) and future time periods are downloaded from the CCAFS website (http://www.ccafs-climate.org). Future time periods include 2021-2040 (2030s) and 2041-2060 (2050s). 2061-2080 (2070s), 2071-2090 (2080s), select RCP2.6 issued by IPCC (low greenhouse gas emission scenarios), RCP4.5 (low greenhouse gas emission scenarios), RCP8.5 (greenhouse gas emissions higher scenarios). Three typical concentration paths are used as future climate scenarios (Zhou et al., 2014; Lin \& Zhou, 2015; Ying et al., 2016).

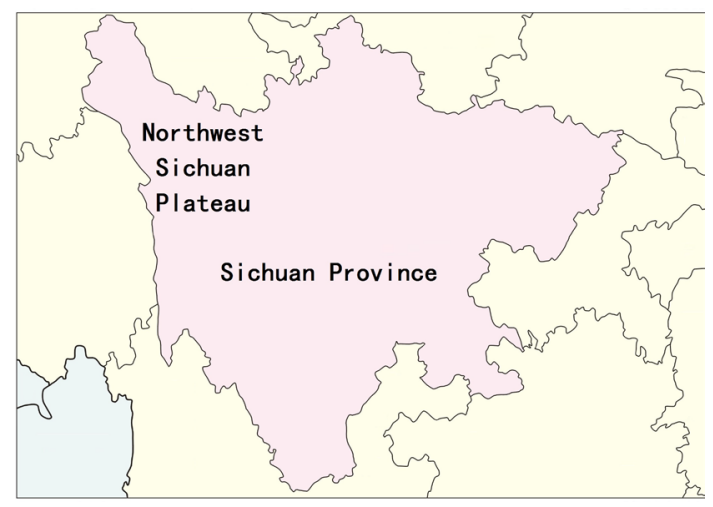

Figure 1. Schematic diagram of northwest Sichuan Plateau in Sichuan Province, China. 
Our research area includes Ganzi Tibetan Autonomous Prefecture and Aba Tibetan and Qiang Autonomous Prefecture in Sichuan Province, China. We used ARCGIS software to extract 19 bioclimatic data from the reference period and future time of the study area. We selected $\geq 0{ }^{\circ} \mathrm{C}$ accumulated temperature, dryness and wetness index as the climate indicator data. We also selected altitude data as a topographic factor. The spatial resolution of the above data is unified to 2.5 arc-minutes. Each grid cell is roughly equivalent to $4.5 \mathrm{~km}^{2}$ (Appendix Table A1).

\subsection{Construction of the Niche Model}

The geographical distribution of cultivated grasses was predicted using MaxEnt version 3.4.1 software (Hijmans et al., 2005; Phillips et al., 2006). The simulation results were calculated, graded, and post-processed using ARCGIS 10.2 software.

1) Based on the training subset ( $75 \%$ of the total data set randomly selected as the training subset) to construct the maximum entropy model of the cultivated grass in the study area; the remaining $25 \%$ of the data constructed by the non-participating model is used as the test subset. (Testing data) to verify the model, the number of training repetitions of the model is set to 10 times.

2) The model is tested by ROC curve. The method is based on the area under curve (AUC). The AUC threshold is divided into: $0.5 \leq$ AUC $<0.6$ simulation failure, $0.6 \leq \mathrm{AUC}<0.7$ accuracy is poor, $0.7 \leq \mathrm{AUC}<0.8$ simulation results in general, $0.8 \leq$ AUC $<0.9$ simulation results are good, $0.9 \leq$ AUC $<1$ simulation results are excellent.

3) Using the Jackknife test to test the environmental factor contribution rate of the geographical distribution of cultivated grasses in the study area. The method initially assumes that the species is evenly distributed, and creates a series of models by sequentially using or excluding a variable, thereby presuming the dominant environmental factors affecting the geographical distribution of cultivated grasses.

4) Input environmental variables in the contemporary prediction of cultivated grasses include 19 bioclimatic data, $\geq 0^{\circ} \mathrm{C}$ accumulated temperature, dryness index, wetness index, and altitude data, with a total of 23 environmental variables to study the leading environmental factor indicators, which is convenient for guiding the planting base. Construction; the prediction of the geographical distribution of the base age and future climate scenarios when inputting 19 bioclimatic data corresponding to the era to focus on the impact of climate change on the geographical distribution of cultivated grasses.

\subsection{Prediction and Division of Suitable Areas}

The highest ROC value is selected from the MaxEnt software analysis results as the final result, and converted into Raster format by importing ARCGIS 10.2 software. The MaxEnt model run results calculate the probability $P$ value $(0 \leq P$ $<1$ ) of the species in each grid cell. The smaller the $P$ value, the smaller the probability that the species will appear in this unit, and vice versa. This study re- 
fers to the relevant scholars' research (Kong et al., 2019; Zhou et al., 2015), combined with the actual selection of the grassland planting base in Northwest Sichuan, based on the MaxEnt software automatically generated Equal training sensitivity and specificity threshold (contemporary is 0.298 ), according to the natural halving method. The suitable area for perennial cultivation of grass is divided into four levels: 1$)$ unsuitable area, $P<0.298$; 2) low suitable area, $0.298 \leq$ $P<0.526 ; 3$ medium suitable area, $0.526 \leq P<0.754 ; 4 P \geq 0.754$. Highly suitable area. Based on this classification, ARCGIS 10.2 software was used to make a zoning map for the potential distribution of perennial cultivated grasses.

Corresponding to three greenhouse gas emission scenarios, each successive generation receives four consecutive probability distribution maps. The analysis methods for the base period and specific future years are as follows: 1) Refer to the IPCC Fourth Assessment Report for the assessment of the threshold of assessment possibility (Duan \& Zhou, 2011), the climate suitable for grading criteria: $P<0.05$ is the climate unsuitable area; $0.05 \leq P<0.33$ is a suitable climate zone; $0.33 \leq P<0.66$ is a suitable climate zone; $P \geq 0.66$ is a high climate suitable zone. A total of 13 distribution probability maps were obtained from the baseline and the three climate scenarios in the next four eras, and then the predicted area change analysis was carried out by time and different emission climate scenarios; 2) In combination with the perennial cultivation of grasses in the Northwest Sichuan Plateau, $P \geq 0.33$. The distribution probability values (high-appropriate area and medium-suitable area) are set as "appropriate areas", and the 13-distribution probability maps of the benchmark and specific future years are converted into the Presence/Absence binary raster map; 3 the benchmarks to be obtained and the next 4 . In the different climate scenarios of the era, the binary raster map is superimposed, and the highest value of a single pixel is 4 , and the unit pixel value $\geq 3$ is the climate-appropriate area, thus obtaining the perennial cultivated grass distribution in the Northwest Sichuan Plateau under the climate change background. The relatively stable climate-appropriate zone refers to the region where the species distribution is suitable under the baseline and the future three greenhouse gas emission scenarios, that is, it is less affected by climate change.

\section{Results and Analysis}

\subsection{Applicability Analysis of the Model}

This paper uses the AUC value to test the applicability of the model. The results show that the AUC values of the predicted age model training set and the verification set data are 0.925 and 0.929 , respectively, which are higher than 0.5 of the stochastic model, and the simulation results are all excellent. This shows that the accuracy of the constructed model is extremely high and can be used in the Northwest Sichuan Plateau. The suitability of perennial cultivated grasses is predicted. Table 1 shows the AUC values of the MaxEnt model of perennial cultivated grasses distributed in the Northwest Sichuan Plateau under the predicted 
Table 1. Predicted and AUC values of the MaxEnt model under future climate change scenarios.

\begin{tabular}{ccc}
\hline Climate scenario & Training data & Test data \\
\hline Forecast year & 0.925 & 0.929 \\
Current & 0.9034 & 0.9567 \\
2030s (Rcp2.6) & 0.885 & 0.9386 \\
2030s (Rcp4.5) & 0.8503 & 0.9047 \\
2030s (Rcp8.5) & 0.8529 & 0.9064 \\
2050s (Rcp2.6) & 0.855 & 0.9386 \\
2050s (Rcp4.5) & 0.851 & 0.9147 \\
2050s (Rcp8.5) & 0.8501 & 0.9064 \\
2070s (Rcp2.6) & 0.868 & 0.9191 \\
2070s (Rcp4.5) & 0.8688 & 0.9295 \\
2070s (Rcp8.5) & 0.895 & 0.9491 \\
2080s (Rcp2.6) & 0.887 & 0.9426 \\
2080s (Rcp4.5) & 0.85 & 0.9088 \\
2080s (Rcp8.5) & 0.9012 & 0.9533 \\
\hline
\end{tabular}

age and different climate change scenarios. It can be seen that the AUC values of the constructed model training data are all greater than 0.85 , and the test data AUC values are greater than 0.9. This shows that the accuracy of the model built in this study is high and the prediction results are available.

\subsection{Analysis of Contemporary Simulation Prediction Results}

\subsubsection{Main Environmental Variables Affecting the Distribution of Perennial Cultivated Grasses}

In this study, based on the results of the model, the cumulative contribution rate of different environmental impact factors is to the potential distribution of perennial cultivated grasses. The wetness index, $\geq 0^{\circ} \mathrm{C}$ accumulated temperature, and isothermal contribution rate were the highest, both exceeding $15 \%$. The cumulative contribution rate of the above three factors reached $74.8 \%$, indicating that they are the main climatic factors that determine the distribution of perennial cultivated grasses in the Northwest Sichuan Plateau; the warmest month, the highest temperature, the dryness index, the altitude, the annual average temperature range, etc. The contribution rate of each factor is small, but it is still important for predicting the distribution of perennial cultivated grasses under the harsh and varied plateau ecological environment. The cumulative contribution of these seven factors is to the distribution of perennial cultivated grasses in the Northwest Sichuan Plateau. The rate is over $90 \%$.

In the study, the correlation between the seven factors was calculated using the spearman correlation coefficient method to eliminate the influence of collinearity on the model modeling process and the interpretation of the results. If 
the absolute value of the correlation coefficient between the factors is greater than 0.8 , the correlation between the two is extremely significant (Hijmans et al., 2005; Lemke et al., 2011). It can be seen from Table 2 that except for the three environmental factors of altitude, warmest month maximum temperature and dryness index, the correlation coefficients between the other factors have not reached a very significant correlation level. Therefore, the study determines the wetness index. The $\geq 0^{\circ} \mathrm{C}$ accumulated temperature, isothermal and annual mean temperature range were used as the dominant factors affecting the distribution of perennial cultivated grasses in the Northwest Sichuan Plateau. On this basis, the maximum entropy model was reconstructed and the accuracy of the simulation results was evaluated. From this we can get the optimal prediction model. The AUC values of the training set and the validation set data of the reconstructed model are 0.932 and 0.946 , respectively, which is better than the pre-reconstruction model, and the simulation results are all excellent. The wetting index, $\geq 0{ }^{\circ} \mathrm{C}$ accumulated temperature, isothermal contribution rate. The maximum is more than $15 \%$. The contribution rate of each factor is humid index $>$ accumulated temperature $\left(\geq 0^{\circ} \mathrm{C}\right)>$ isothermal $>$ annual average temperature change, and the contribution rate of single index of wetting index and $\geq 0{ }^{\circ} \mathrm{C}$ accumulated temperature. Both are greater than $25 \%$, and the cumulative proportion is $73.2 \%$, indicating that the dominant environmental factors affecting the distribution of perennial cultivated grasses in the Northwest Sichuan Plateau are the result of comprehensive determination of various climatic conditions.

Figure 2 is a feedback curve between the dominant environment variable and the distribution probability plotted by the MaxEnt model, which can reflect the range of values of environmental variables under different thresholds. Referring to the classification method of 1.4 , this paper uses $0.298(29.8 \%)$ as the threshold to divide the environmental variables that affect the distribution of perennial cultivated grasses in the Northwest Sichuan Plateau. As a warm plant, the suitable climatic conditions for gramineous forage must have certain water and heat conditions. The results show that the existence probability of perennial cultivated grass in the range of 48.6 - 90 is continuously rising; the accumulated temperature the suitable value range is $1118.2^{\circ} \mathrm{C}-2350.5^{\circ} \mathrm{C}$, the optimum value

Table 2. Pair-wise spearman's correlation coefficients of environmental variables.

\begin{tabular}{ccccccc}
\hline Environmental Variables & Alt & Att0 & Ai & Im & Bio3 & Bio5 \\
\hline Att0 & $-0.82^{* *}$ & & & & \\
Ai & $-0.46^{*}$ & $0.81^{\star *}$ & & & \\
Im & 0.02 & $-0.28^{*}$ & $-0.29^{*}$ & & \\
Bio3 & $-0.21^{*}$ & $0.39^{*}$ & $0.11^{*}$ & 0.02 & \\
Bio5 & $-0.90^{* *}$ & $0.83^{* *}$ & $0.71^{\star *}$ & 0.01 & $0.47^{*}$ \\
Bio7 & $0.32^{*}$ & $-0.43^{*}$ & $-0.29^{*}$ & $0.44^{\star}$ & $-0.17^{\star}$ & $-0.19^{*}$ \\
\hline
\end{tabular}

Notes: The environmental variables are described in appendix; ${ }^{*}$ indicates Significant correlation at $\alpha=$ $0.05 ;{ }^{*}$ indicates significant correlation at $\alpha=0.01$. 

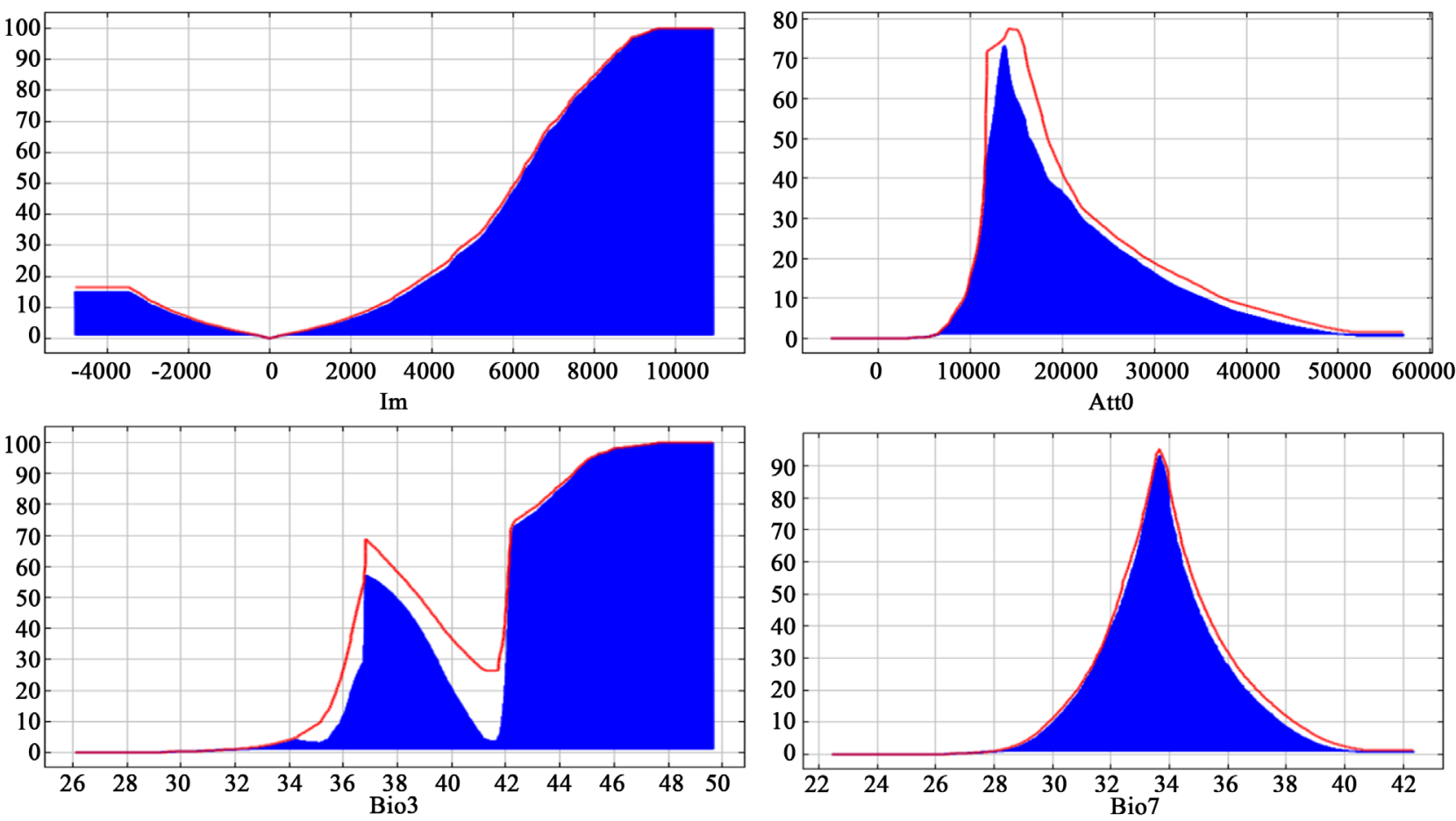

Figure 2. Feedback curves of perennial cultivated grass to environmental variables in MaxEnt model.

is $1497.8^{\circ} \mathrm{C}$, and the distribution probability increases sharply with the increase of heat at $1118.2^{\circ} \mathrm{C}-1497.8^{\circ} \mathrm{C}$. At $1497.8^{\circ} \mathrm{C}-2350.5^{\circ} \mathrm{C}$, the distribution probability decreases sharply with the increase of heat; the isothermal suitability value $36.1 \%-40.9 \%$ or $>41.7 \%$, optimum value $36.8 \%, 41.7 \%-42.0 \%$. The probability of perennial cultivated grasses is continuously rising; the appropriate value of annual average temperature range is $31.4^{\circ} \mathrm{C}-36.2^{\circ} \mathrm{C}$, and the optimum value is $33.7^{\circ} \mathrm{C}$, at $31.4^{\circ} \mathrm{C}-33.7^{\circ} \mathrm{C}$, the probability of distribution increases sharply with the increase of the year, and the probability of distribution increases sharply with the increase of the year at $33.7^{\circ} \mathrm{C}-36.2^{\circ} \mathrm{C}$. Using the results of the suitable area of the model output, using the spatial analysis method of ARCGIS, the average elevation value of the suitable area is $2700 \mathrm{~m}$, the average elevation of the concentrated distribution area is $3530 \mathrm{~m}$, and the highest elevation of the suitable area is $<4859 \mathrm{~m}$ (Table 3 ).

\subsubsection{Prediction of Geographical Distribution of Contemporary} Perennial Cultivated Grasses in the Northwest Plateau of Sichuan

According to the results of four dominant factor variables and the distribution data of cultivated grasses in the Northwest Sichuan Plateau, the results of the maximum entropy model were constructed according to the four criteria of high-adapted area, middle-aged area, low-adapted area and unsuitable area. The prediction results show that the high-adapted area is mainly located in the meadow grassland belt of pure pastoral area in Aba Prefecture and Ganzi Prefecture; the middle-low-estate area is distributed around the edge of the high-adapted area, mainly located in the semi-agricultural and semi-agricultural district, and 
Table 3. Appropriate range value of environmental variables for perennial cultivated grass distribution in northwest Sichuan Plateau.

\begin{tabular}{ccc}
\hline Environmental Variables (Unit) & Suitable Range & Optimum Value \\
\hline Im & $>48.6$ & - \\
$\operatorname{Att} 0\left({ }^{\circ} \mathrm{C}\right)$ & $1118.2-2350.5$ & 1497.8 \\
$\operatorname{Bio} 3(\%)$ & $36.1-40.9$ or $>41.7$ & 36.8 \\
$\operatorname{Bio7}\left({ }^{\circ} \mathrm{C}\right)$ & $31.4-36.2$ & 33.7 \\
Alt $(\mathrm{m})$ & $2700-4859$ & 3530 \\
\hline
\end{tabular}

Notes: The environmental variables are described in Appendix.

the low-suitable area is mainly located in the farmland. Areas such as alpine meadow pastures in districts and counties, the unsuitable areas are mainly located in the permanent snow cover above the alpine snow line, desert, bare rock, bare land and low altitude and valley areas of agricultural districts. The locations of the artificially planted pasture bases obtained from the current survey are all located in the Gaoshen area except for the Bamei Ranch in Daofu County. It is found that the underlying nature of these planting bases is dominated by meadow pastures. Fuxian Bamei Ranch has a relatively warm climate due to special terrain. It is mainly planted with Onobrychis viciaefolia Scop and Hordeum vulgare Linn. var. nudum Hook.f, and a small amount of other grasses are planted. The base is located in a low-yielding area of perennial cultivated grass.

The perennial cultivated grass has a total area of $5.45 \times 10^{4} \mathrm{~km}^{2}$, accounting for $21.7 \%$ of the total area of the Sichuan Plateau. Among them, the areas of high, medium and low suitable areas are $0.42 \times 10^{4} \mathrm{~km}^{2}, 1.69 \times 10^{4} \mathrm{~km}^{2}$ and 3.34 $\times 10^{4} \mathrm{~km}^{2}$, respectively accounting for $1.7 \%, 6.7 \%$ and $13.3 \%$ of the total area of the Northwest Plateau.

\subsection{Prediction of Potential Distribution of Perennial Cultivated Grasses Under Climate Change Scenarios}

\subsubsection{Changes in Climate-Appropriate Area of Perennial Cultivated Grasses under Climate Change}

In the context of climate change, this study predicts climate-appropriate areas under three greenhouse gas emissions scenarios in the contemporary and future four years (Table 4). The results show that the area of suitable areas with high, medium and low climates is increasing compared with the baseline climate, and the increase of climate suitable area is characterized by suitable climate zone $>$ climate high suitable zone $>$ low climate suitable zone.

1) Change of high climate suitable area: By 2080s, the area of high climate suitable area will increase to $1.4 \times 10^{4} \mathrm{~km}^{2}, 1.3 \times 10^{4} \mathrm{~km}^{2}$ and $1.4 \times 10^{4} \mathrm{~km}^{2}$ under the RCP2.6, RCP4.5 and RCP 8.5 scenarios, respectively. They increased by $47.4 \%, 32.6 \%$, and $47.4 \%$, respectively.

2) Appropriate zone change in climate: By 2080 s, the suitable area of the climate will increase to $5.3 \times 10^{4} \mathrm{~km}^{2}, 5.1 \times 10^{4} \mathrm{~km}^{2}$ and $5.7 \times 10^{4} \mathrm{~km}^{2}$ under the 
Table 4. Prediction of potential suitable areas for perennial cultivated grass baseline and different climate scenarios in future years.

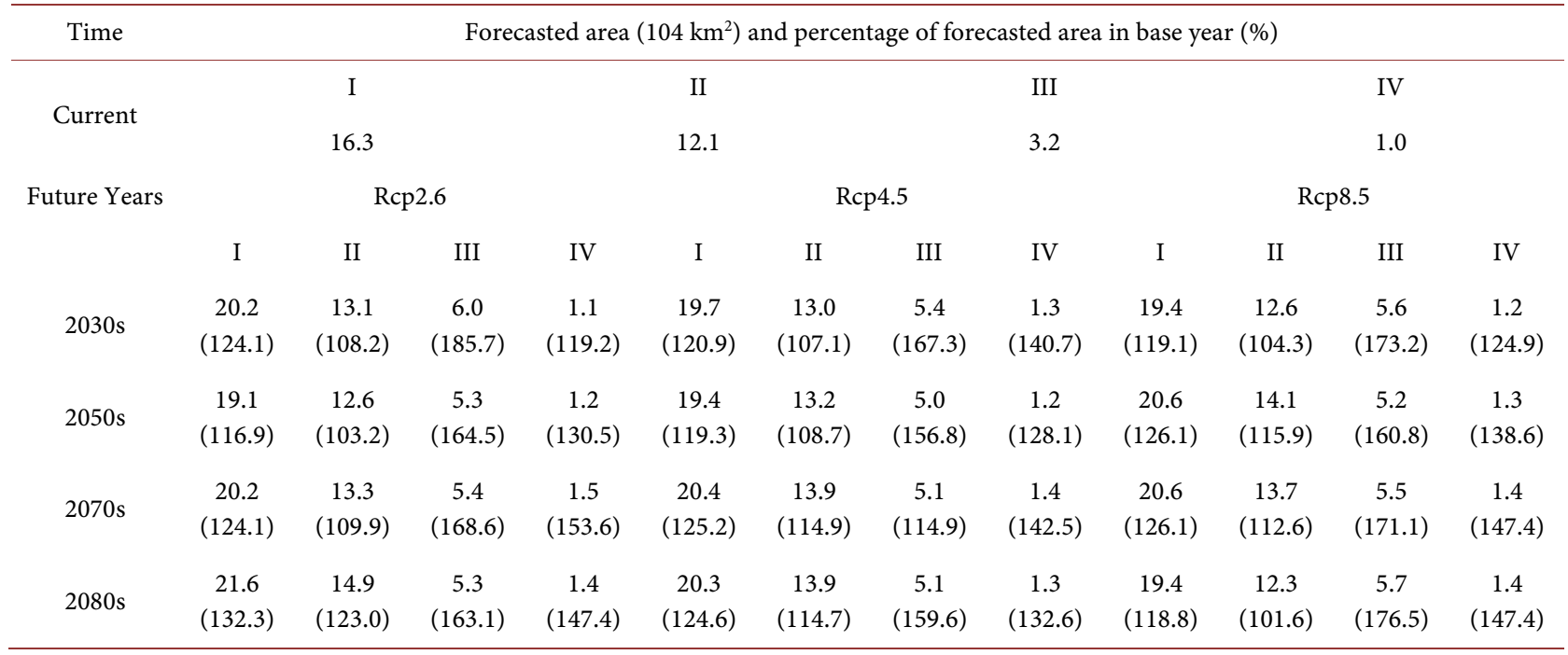

Notes: I. Total climatic suitable area; II. Low climatic adaptable area; III. Medium climate suitable area; IV. High climate suitable area; Forecasted area percentage of forecasted area in base year.

RCP2.6, RCP4.5 and RCP8.5 scenarios, respectively. They increased by $63.1 \%$, $59.6 \%$, and $76.5 \%$, respectively.

3) Change of low climate suitable area: By 2080s, the area of low climate suitable area will increase to $14.9 \times 10^{4} \mathrm{~km}^{2}, 13.9 \times 10^{4} \mathrm{~km}^{2}$ and $12.3 \times 10^{4} \mathrm{~km}^{2}$ respectively under the scenarios of RCP2.6, RCP4.5 and RCP8.5. They increased by $23.0 \%, 14.7 \%$, and $1.6 \%$, respectively.

\subsubsection{Prediction of the Potential Distribution of Perennial Cultivated Grasses in the Context of Climate Change}

In the next four years, the area of perennial cultivated grasses in the Northwest Sichuan Plateau will be significantly increased, and the climate-appropriate area will increase by $52.7 \%\left(6.0 \times 10^{4} \mathrm{~km}^{2}, 2030 \mathrm{~s}, 2080 \mathrm{~s}\right)-65.5 \%$. $\left(6.5 \times 10^{4} \mathrm{~km}^{2}\right.$, $2050 \mathrm{~s}$ ), accounting for $24.7 \%$ (2030s, 2080s) to $26.7 \%$ (2050s) of the total area of the study area; the average altitude of the climate-friendly area has an increasing trend, and the average altitude increase is In the range of $212.4 \mathrm{~m}(3690.0 \mathrm{~m}$, 2050s) - $252.0 \mathrm{~m}$ (3729.6 m, 2080s), the highest altitude is $4989 \mathrm{~m}$, an increase of $131 \mathrm{~m}$.

The perennial cultivated grasses are relatively stable and suitable for climatically concentrated areas in Zoige, Hongyuan, Aba County, northern Sichuan, northern Songpan, Shiqu, Seda, Ganzi, Dege, Litang, Luhu, Ba, Meadow grassland area in Tang and other places. In addition, there are also a small number of alpine meadow pastures in the black water, Malcom, Daofu, Yajiang, Baiyu, Daocheng, Xiangcheng and other counties of the alpine valley. The artificially cultivated pasture bases currently surveyed are all located in the climate-friendly area. According to the model prediction results, the contemporary climate-friendly areas of alpine meadow pastures in Wenchuan, Lixian, Maoxian, Xiaojin, Danba, 
Kangding, Luding, Jiulong, Derong, and southern townships in the Southwest Sichuan Plateau will disappear completely. The climatically suitable areas of perennial cultivated grasses have a tendency to expand into alpine grasslands, alpine deserts and currently permafrost zones.

\section{Discussion}

\subsection{Accuracy of Perennial Cultivated Grass Simulation}

In this study, the maximum entropy model was used to simulate the potential distribution of perennial cultivated grasses in the Northwest Sichuan Plateau based on the selected dominant climatic factors. The simulation accuracy reached an excellent level (AUC $=0.946)$. The results of the model simulation showed that the artificial pasture planting bases obtained in the survey are all located within the suitable area, indicating that the potential geographical distribution prediction results of the model simulation are highly reliable.

The number of species distribution point data will affect the effect of model simulation. The study shows that (David \& Townsend, 2002; Wisz et al., 2008) for the construction of the maximum entropy model of small sample size distribution, when the number of training samples is higher than 30 , the prediction result of the maximum entropy model is stable; when the training sample size is 10 , the maximum prediction success rate of the model is less than $90 \%$; when the training sample size is 50 , the prediction success rate of the model can be maximized. The number of training samples used to construct the maximum entropy model is 35 . The stability and simulation results of the maximum entropy model are good, which can be used to predict the distribution of perennial cultivated grasses in the Northwest Sichuan Plateau.

\subsection{Main Environmental Variables Affecting the Distribution of Perennial Cultivated Grasses in the Northwest Sichuan Plateau}

The main factors limiting the geographical distribution of perennial cultivated grasses include the cold tolerance of plants, the heat and water conditions required to complete the growth period. The results of this study showed that the main climatic factors affecting the distribution of perennial cultivated grasses were the wetness index $(\mathrm{Im}), \geq 0^{\circ} \mathrm{C}$ accumulated temperature (Att0), isothermal (Bio3), annual mean temperature range (Bio7), and the wetness index and $\geq 0$. The contribution rate of ${ }^{\circ} \mathrm{C}$ accumulated temperature to the model exceeded $25 \%$. It indicates that the perennial cultivated grass can grow in a certain area, which is not determined by a single climatic condition, but is the result of comprehensive determination of various climatic conditions. Among them, the water condition is the main environmental factor, and the wetting index characterizes the regional water budget, which can be used to determine the amount of water (Thornthwaite, 1948). Wu (1988) pointed out that the grasses of grasses can only use the moisture of the surface layer of the soil because of the small 
roots. They need more water than the legumes, and the water shortage is quickly withered. In addition, the heat condition is also one of the environmental factors affecting the distribution of perennial cultivated grasses. Due to the harsh environmental conditions in the grassland area of the Northwest Sichuan Plateau, the forage growing season is shorter (Zhang et al., 2018) (the growth period is from May to October, the flowering period is from July to September), and only after a certain heat condition can the perennial cultivation of grass be Using this short season to complete the growth period, the suitable value of the accumulated temperature required for the growth of perennial cultivated grasses in this study ranged from $1497.8^{\circ} \mathrm{C}$ to $2350.5^{\circ} \mathrm{C} \cdot \mathrm{d}$, which is suitable for the thermal conditions of perennial cultivated grasses in Qinghai, which was studied by Zhou et al. (2018). The range of values $\left(1200^{\circ} \mathrm{C}-2200^{\circ} \mathrm{C} \cdot \mathrm{d}\right)$ is not much different; the isothermal (Bio3) and annual mean temperature range (Bio7) determine whether the perennial cultivated grass can survive the winter. Chen et al. (2016) also studied the simulation studies of Carex moorcroftii Falc. Ex Boott on Sinopodophyllum hexandrum, and Liu et al. (2018) and Guo et al. (2014) studied Sinopodophyllum hexandrum and Carex moorcroftii Falc. Ex Boott, respectively. It is shown that temperature affects the growth and reproduction of these plants and thus determines their geographical distribution. Therefore, these environmental variables are the main factors affecting the distribution of perennial cultivated grasses.

\subsection{Prediction of the Distribution of Perennial Cultivated Grasses in the Northwest Sichuan Plateau under the Background of Climate Change}

According to the Sichuan Provincial Climate Change Monitoring Bulletin, the annual average temperature and annual precipitation of the Sichuan Plateau in the period from 1961 to 2017 were $0.23^{\circ} \mathrm{C} \cdot(10 \mathrm{a})^{-1}$ and $8.1 \mathrm{~mm} \cdot(10 \mathrm{a})^{-1}$, respectively. ( $\mathrm{Hu}$ et al., 2015a), the average annual temperature trend of the Qinghai-Tibet Plateau in $2006-2100$ is $0.26^{\circ} \mathrm{C} \cdot(10 \mathrm{a})^{-1}$, and the temperature increase in high altitude areas is relatively large. The temperature increase in low-altitude areas is relatively small, and the precipitation increases slightly. The average trend is $1.15 \% \cdot(10 a)^{-1}$. The results of this study show that the reference age is 2080s. As the climate warms, the potential distribution of perennial cultivated grasses in the Northwest Sichuan Plateau will increase. The perennial cultivated grasses of alpine meadow pastures in some agricultural areas in the southern part of the plateau. The climate-appropriate zone will disappear completely, and the average and altitude of the perennial cultivated grasses will increase. Wu \& $\mathrm{Lu}$ (2009) pointed out that under climate change, the suitable climate distribution range of the alpine meadow in the Qinghai-Tibet Plateau will be reduced, and the new suitable climate distribution will increase. The alpine meadow will mainly expand to the current alpine grassland and alpine desert distribution area. And, the precipitation in the alpine steppe and the alpine desert region will show an increasing trend, and the degree of wetness will also increase. Hu et al. 
(2015a) pointed out that compared with the current period and 2080s, the distribution of Stipa breviflora in the Qinghai-Tibet Plateau, the local expansion has also retreated, the probability of occurrence of Stipa grandis in some areas will increase. In addition, climate warming will cause low-altitude plants to migrate to high-altitude areas. Lenoir (Lenoir et al., 2008) studies of alpine plants around the world have found that climate warming leads to a significant increase in the average altitude of the species, rising every ten years, rising $29 \mathrm{~m}$ every ten years. According to the research results of 2.3, perennial grasses in the Northwest Sichuan Plateau are mainly distributed in alpine meadow pastures. Therefore, under the conditions of climate change, due to the increase of alpine meadow area and the increase of altitude, the alpine grassland and alpine desert in the Northwest Sichuan Plateau. The area will probably be suitable for the growth of perennial cultivated grasses, and the overall climate-friendly area will show an increasing trend.

This study predicted the distribution of perennial cultivated grasses in the Northwest Sichuan Plateau according to the baseline and climate change scenarios. Due to the uncertainty and periodicity of climate warming, this will affect the prediction results of the model (Yi et al., 2016), using MaxEnt alone. Model prediction also increases the uncertainty of the prediction results; secondly, the sample size and geographic location accuracy of the species collection will also have an impact on the prediction accuracy of the model. In addition, in addition to climatic factors, factors affecting species distribution include other biotic and abiotic factors, such as inter-species competition, plant adaptation to the environment, and topography, soil properties, etc., underlying surface caused by human factors. Changes in environmental factors such as changes in nature and meadow degradation caused by overgrazing, this study has not yet entered the model as a variable for prediction, which will affect the accuracy of the prediction results, so there is room for further improvement in the simulation results.

\section{Conclusion}

Using the maximum entropy model to predict the potential distribution of perennial cultivated grasses in the Northwest Sichuan Plateau, the AUC values of the model training data were all greater than 0.85 , and the AUC values of the test data were all greater than 0.9 , both of which reached good and excellent levels.

The four main climatic factors affecting the distribution of perennial cultivated grasses are the wetness index, $\geq 0^{\circ} \mathrm{C}$ accumulated temperature, isothermal, and annual mean temperature variation. The grassland meadow area in the Northwest Sichuan Plateau is the most suitable area for the distribution of perennial cultivated grasses.

Under the future climate change scenario, from now to the future (2080s), the potential distribution of perennial cultivated grasses in the Northwest Sichuan Plateau will increase, and the altitude will gradually increase.

Perennial cultivated grass is the dominant species for the establishment of ar- 
tificial grassland in the alpine meadow pasture in the Northwest Sichuan Plateau. It plays a vital role in the soil and water conservation, desertification control, modern highland characteristic animal husbandry base construction and ecological balance. The research results can be used as a reference for the construction of the first demonstration zone of ecological civilization in northwest Sichuan and the work of addressing climate change.

\section{Acknowledgements}

This paper is organized by the National Key R \& D Program (2017YFC0504903) \& National Key R \& D Program of China (2017YFC0504903), National Natural Science Foundation of China (41775159), Sichuan Provincial Meteorological Bureau 2018 Research Project (provincial laboratories 2018-emphasis-05-10) Funded by the Sichuan Science and Technology Department Project (2017ZR0281).

\section{Conflicts of Interest}

The authors declare no conflicts of interest regarding the publication of this paper.

\section{References}

Bellard, C., Bertelsmeier, C., Leadley, P., Thuiller, W., \& Courchamp, F. (2012). Impacts of Climate Change on the Future of Biodiversity. Ecology Letters, 15, 365-377. https://doi.org/10.1111/j.1461-0248.2011.01736.x

Chen, J. J., Yan, Y. Y., Cong, R. H. et al. (2016). Research and Estimation of Potential Distribution Area of Stipa breviflora Based on MaxEnt Model in China. Chinese Journal of Grassland, 38, 78-84.

Chen, J. S., Liu, J. L., Zhu, R. F. et al. (2019). Analysis of Bioclimatic Characteristics of Leymus chinensis Distribution Area in China Based on MaxEnt. Acta Agrestia Sinica, $27,35-42$.

Davis, M. B., \& Shaw, R. G. (2001). Shifts and Adaptive Responses to Quaternary Climate Change. Science, 292, 673-679. https://doi.org/10.1126/science.292.5517.673

Department of Animal Husbandry and Veterinary, Ministry of Agriculture, National Animal Husbandry and Veterinary Station (1996). China Grassland Resources (pp. 21-22). Beijing: China Science and Technology Press.

Ding, Y. H., Ren, G. Y., Shi, G. Y. et al. (2006). National Assessment Report on Climate Change: History and Future Trends of Climate Change in China. Progress in Climate Change Research, 1, 3-8.

Dong, C. S. (1986). Grassland Resources in Aba Prefecture, Sichuan Province (pp. 30-35). Agricultural Bureau of Aba Prefecture. Malcom.

Duan, J. Q., \& Zhou, G. S. (2011). Potential Distribution of Rice in China and Its Climatic Characteristics. Acta Ecologica Sinica, 31, 6659-6668.

Duo, J. Z. (1986). Investigation and Division of Agricultural Resources in Ganzi Tibetan Autonomous Prefecture, Sichuan Province-Grassland Resources (pp. 287-302). Kangding: Ganzi Prefecture Grassland Station.

Guo, Y. L., Wei, H. Y., Lu, C. Y. et al. (2014). Prediction of Potential Geographical Distribution of Taoer-7 under Climate Change. Chinese Journal of Plant Ecology, 38, 
249-261.

Hijmans, R. J., Cameron, S. E., Parra, J. L., Jones, P. G., \& Jarvis, A. (2005). Very High Resolution Interpolated Climate Surfaces for Global Land Areas. International Journal of Climatology, 25, 1965-1978. https://doi.org/10.1002/joc.1276

Hong, Y. Z. (1989). Regionalization of Perennial Cultivated Grass Species in China (pp. 158-177). Beijing: China Agricultural Science and Technology Press.

Hu, W., Jiang, D., \& Fan, G. Z. (2015a). Future Climate Change Prediction of the Tibetan Plateau: Results of CMIP5 Mode. Chinese Journal of Atmospheric Sciences, 39, 260-270.

Hu, Z. J., Zhang, Y. L., \& Yu, H. B. (2015b). Simulation of Distribution Pattern of Stipa breviflora on Qinghai-Tibet Plateau Based on MaxEnt Model and GIS. Chinese Journal of Applied Ecology, 26, 505-511.

Kong, W. Z., Li, X. H., \& Zou, H. F. (2019). Optimization of Maximum Entropy Model in Species Distribution Prediction. Chinese Journal of Applied Ecology, 30, 2116-2128.

Lemke, D., Hulme, P. E., Brown, J. A., \& Tadesse, W. (2011). Distribution Modelling of Japanese Honeysuckle (Lonicera japonica). Invasion in the Cumberland Plateau and Mountain Region, USA. Forest Ecology and Management, 262, 139-149. https://doi.org/10.1016/j.foreco.2011.03.014

Lenoir, J., Gégout, J. C., Marquet, P. A. et al. (2008). A Significant Upward Shift in Plant Species Optimum Elevation during the 20th Century. Science, 320, 58-84. https://doi.org/10.1126/science.1156831

Lin, Y. P., \& Zhou, T. J. (2015). Characteristics of Precipitation Structure and Future Changes in East Asia Region Participated in Four Chinese Models of CMIP5 Project. Chinese Journal of Atmospheric Sciences, 39, 338-356.

Liu, W. S., You, J. J., Zeng, W. B. et al. (2018). Prediction of the Geographical Distribution of Carex lasiocarpa under Climate Change. Chinese Journal of Grassland, 40, 43-49.

Ma, Y. B., Yan, W. H., Xu, Z. et al. (2014). Investigation and Collection of Wild Forage Germplasm Resources in Sichuan and Tibetan Areas. Chinese Wild Plant Resources, 33, 36-39.

Pan, C. B., \& Wang, Y. F. (1993). A Comprehensive Report on the Study of Hydrothermal Ecological Characteristics of Cultivated Pasture in the Alpine Pastoral Area of Northwest, Sichuan. Sichuan Grassland, 1, 20-22.

Phillips, S. J., Anderson, R. P., \& Schapire, R. E. (2006). Maximum Entropy Modeling of Species Geographic Distributions. Ecological Modelling, 190, 231-259. https://doi.org/10.1016/j.ecolmodel.2005.03.026

Qin, D. H., \& Stocker, T. (2014). IPCC Fifth Assessment Report The First Working Group Report Highlights Conclusion. Progress in Climate Change Research, 10, 1-6.

Stockwell, D., R. B., \& Townsend Peterson, A. (2002). Effects of Sample Size on Accuracy of Species Distribution Models. Ecological Modelling, 148, 1-13. https://doi.org/10.1016/S0304-3800(01)00388-X

Tang, M. K., Mao, Y. J., Liu, K. Y. et al. (2018). Characteristics of Wetland Flora and Preliminary Survey of Wetland Community in the Northwest Sichuan Plateau. Sichuan Forestry Science and Technology, 39, 71-78.

Thornthwaite, C. (1948). An Approach toward a Rational Classification of Climate. Geographical Review, 38, 55-94. https://doi.org/10.2307/210739

Wang, Y. F., Yang, Z. Y., \& Pan, C. B.(1995). Breeding Report of Chuancao 2 Laomeng. Sichuan Grassland, 1, 19-24.

Wisz, M. S., Hijmans, R. J., Li, J., Peterson, A. T., Graham, C. H., \& Guisan, A. (2008). 
Effects of Sample Size on the Performance of Species Distribution Models. Diversity and Distributions, 14, 763-773. https://doi.org/10.1111/j.1472-4642.2008.00482.x

Wu, G. Y. (1988). Agricultural Meteorological Conditions for Growth and Yield Formation of Grasses (Graphic). China Grassland, 1, 60-64.

Wu, J. G., \& Lu, J. J. (2009). The Potential Impact of Climate Change on the Suitable Climate Distribution of Alpine Meadows on the Qinghai-Tibet Plateau. Journal of Grass Industry, 17, 699-705.

Yang, C. (2016). Suitable Distribution Areas of Stipa Genus in Mongolian Plateau and Qinghai-Tibet Plateau and Their Correlation with Climatic Factors. Yuquan, Inner Mongolia: Inner Mongolia University.

Yi, Y. J., Cheng, X., Yang, Z. F., \& Zhang, S. H. (2016). Maxent Modeling for Predicting the Potential Distribution of Endangered Medicinal Plant (H. Riparia Lour). in Yunnan, China. Ecological Engineering, 92, 260-269. https://doi.org/10.1016/j.ecoleng.2016.04.010

Ying, L., Liu, W., Chen, S. T., \& Shen, Z. Z. (2016). Simulation of Potential Distribution Pattern of Fragrant Wood in Southwest China Based on Maximum Entropy Model under Climate Change Scenarios. Biodiversity, 24, 453-461.

https://doi.org/10.17520/biods.2015246

Yu, S. Q., Lin, X. Z., \& Xu, X. D. (2003). The Interdecadal Oscillation of Temperature in China and Its Future Trends. Meteorological Science and Technology, 3, 136-139.

Zhang, C. B., Bai, S. H., Li, D. X. et al. (2018). Comparative Test Report on Seed Production of Maishen Laomeng. Journal of Grassland and Forage Science, 2, 24-26.

Zhang, G. Y., \& Zhang, L. X. (2013). Investigation on the Ecological Environment of the Plateau in Northwest Sichua-Taking Aba Prefecture as an Example. Grass and livestock, 5, 54-57.

Zhang, H. J. (2014). Study on the Characteristics of Climate Change in the Northwest Plateau of Sichuan Province. Journal of Southwest University (Natural Science), 36, 148-156.

Zhang, J. B. (2007). Genetic Diversity of Elymus nutans in the Northwest Sichuan Plateau. Ya'an: Sichuan Agricultural University.

Zhang, L. (2015). Application of MAXENT Maximum Entropy Model in Predicting the Potential Distribution Range of Species. Bulletin of Biology, 50, 9-12.

Zhou, B. R., Han, B. H., Li, S. X. et al. (2018). Study on Climate Suitability of Perennial Cultivated Pasture in Qinghai Province. Journal of Grassland, 26, 591-601.

Zhou, X., Li, Q. Q., Sun, X. B., \& Wei, M. (2014). Simulation and Prediction of Temperature in China by BCC_CSM1.1 Mode. Quarterly Journal of Applied Meteorology, 25, 95-106.

Zhou, Y., Li, Y., \& Wang, Y. M. (2015). Prediction of the Distribution of Suitable Areas of Traditional Chinese Medicine Rhubarb Plants under the Background of Climate Change. Chinese Traditional Medicine, 38, 467-472. 


\section{Appendix}

Table A1. Evaluation index of potential suitable areas for cultivating Gramineae Herbage in Northwest Sichuan Plateau.

\begin{tabular}{|c|c|c|}
\hline Variables and Description & Unit & Significance of variables \\
\hline Bio1 $=$ Annual Mean Temperature & ${ }^{\circ} \mathrm{C}$ & Reflecting the average temperature variability \\
\hline Bio2 $=$ Mean Daily Diurnal Range & ${ }^{\circ} \mathrm{C}$ & Reflecting the temperature difference \\
\hline Bio3 $=$ Isothermality $(\mathrm{Bio} 2 / \mathrm{bi} 07)(\times 100)$ & $\%$ & Reflecting the temperature difference \\
\hline Bio4 $=$ Temperature Seasonality (standard deviation $\times 100)$ & ${ }^{\circ} \mathrm{C}$ & Reflecting the average temperature variability \\
\hline Bio5 $=$ Maximum Temperature of Warmest Month & ${ }^{\circ} \mathrm{C}$ & Reflecting extreme temperature effects \\
\hline Bio6 $=$ Minimum Temperature of the Coldest Month & ${ }^{\circ} \mathrm{C}$ & Reflecting extreme temperature effects \\
\hline Bio7 $=$ Temperature Annual Range (bio5 - bio6) & ${ }^{\circ} \mathrm{C}$ & Reflecting the temperature difference \\
\hline Bio8 = Mean Temperature of Wettest Quarter & ${ }^{\circ} \mathrm{C}$ & Reflect whether the water heat is synchronized \\
\hline Bio9 = Mean Temperature of Driest Quarter & ${ }^{\circ} \mathrm{C}$ & Reflect whether the water heat is synchronized \\
\hline Bio10 $=$ Mean Temperature of Warmest Quarter & ${ }^{\circ} \mathrm{C}$ & Reflecting extreme temperature effects \\
\hline Bio11 $=$ Mean Temperature of Coldest Quarter & ${ }^{\circ} \mathrm{C}$ & Reflecting extreme temperature effects \\
\hline Bio12 $=$ Annual Precipitation & $\mathrm{mm}$ & Reflecting the characteristics of water resources \\
\hline Bio13 = Precipitation of Wettest Monthly & $\mathrm{mm}$ & Reacting extreme moisture conditions \\
\hline Bio14 $=$ Precipitation of Driest Monthly & $\mathrm{mm}$ & Reacting extreme moisture conditions \\
\hline Bio15 = Precipitation Seasonality (Coefficient of variation) & $\%$ & Reflect precipitation and seasonal distribution \\
\hline Bio16 $=$ Precipitation of Wettest Quarter & $\mathrm{mm}$ & Reacting extreme moisture conditions \\
\hline Bio17 = Precipitation of Driest Quarter & $\mathrm{mm}$ & Reacting extreme moisture conditions \\
\hline Bio18 $=$ Precipitation of Warmest Quarter & $\mathrm{mm}$ & Reflect whether the water heat is synchronized \\
\hline Bio19 = Precipitation of Coldest Quarter & $\mathrm{mm}$ & Reflect whether the water heat is synchronized \\
\hline Alt $=$ Altitude & $\mathrm{m}$ & Reflecting altitude differences \\
\hline Att $0=\geq 0^{\circ} \mathrm{C}$ Accumulated Temperature $\left(\geq 0^{\circ} \mathrm{C} \cdot \mathrm{d}, \times 10\right)$ & ${ }^{\circ} \mathrm{C}$ & Reaction heat resource characteristics \\
\hline $\mathrm{A} i=$ Aridity Index $(\times 1000)$ & - & Reaction climate dryness and humidity \\
\hline $\operatorname{Im}=$ Humid Index $(\times 100)$ & - & Reaction climate dryness and humidity \\
\hline
\end{tabular}

